\title{
Front Matter: Volume 7539
}

, "Front Matter: Volume 7539," Proc. SPIE 7539, Intelligent Robots and Computer Vision XXVII: Algorithms and Techniques, 753901 (18 January 2010); doi: 10.1117/12.852291

SPIE Event: IS\&T/SPIE Electronic Imaging, 2010, San Jose, California, United SPIE. States 
PROCEEDINGS

IS\&T / SPIE

Electronic

Imaging

SCIENCE AND TECHNOLOGY

\section{Intelligent Robots and Computer Vision XXVII: Algorithms and Techniques}

David P. Casasent

Ernest L. Hall

Juha Röning

Editors

18-19 January 2010

San Jose, California, United States

Sponsored and Published by

IS\&T-The Society for Imaging Science and Technology

SPIE 
The papers included in this volume were part of the technical conference cited on the cover and title page. Papers were selected and subject to review by the editors and conference program committee. Some conference presentations may not be available for publication. The papers published in these proceedings reflect the work and thoughts of the authors and are published herein as submitted. The publishers are not responsible for the validity of the information or for any outcomes resulting from reliance thereon.

Please use the following format to cite material from this book:

Author(s), "Title of Paper," in Intelligent Robots and Computer Vision XXVII: Algorithms and Techniques, edited by David P. Casasent, Ernest L. Hall, Juha Röning, Proceedings of SPIE-IS\&T Electronic Imaging, SPIE Vol. 7539, Article CID Number (2010).

ISSN 0277-786X

ISBN 9780819479327

Copublished by

SPIE

P.O. Box 10, Bellingham, Washington 98227-0010 USA

Telephone +1 3606763290 (Pacific Time) · Fax +1 3606471445

SPIE.org

and

IS\&T-The Society for Imaging Science and Technology

7003 Kilworth Lane, Springfield, Virginia, 22151 USA

Telephone +1 7036429090 (Eastern Time) · Fax +1 7036429094

imaging.org

Copyright (C) 2010, Society of Photo-Optical Instrumentation Engineers and The Society for Imaging Science and Technology.

Copying of material in this book for internal or personal use, or for the internal or personal use of specific clients, beyond the fair use provisions granted by the U.S. Copyright Law is authorized by the publishers subject to payment of copying fees. The Transactional Reporting Service base fee for this volume is $\$ 18.00$ per article (or portion thereof), which should be paid directly to the Copyright Clearance Center (CCC), 222 Rosewood Drive, Danvers, MA 01923. Payment may also be made electronically through CCC Online at copyright.com. Other copying for republication, resale, advertising or promotion, or any form of systematic or multiple reproduction of any material in this book is prohibited except with permission in writing from the publisher. The CCC fee code is $0277-786 \mathrm{X} / 10 / \$ 18.00$.

Printed in the United States of America.

Paper Numbering: Proceedings of SPIE follow an e-First publication model, with papers published first online and then in print and on CD-ROM. Papers are published as they are submitted and meet publication criteria. A unique, consistent, permanent citation identifier (CID) number is assigned to each article at the time of the first publication. Utilization of CIDs allows articles to be fully citable as soon they are published online, and connects the same identifier to all online, print, and electronic versions of the publication. SPIE uses a six-digit CID article numbering system in which:

- The first four digits correspond to the SPIE volume number.

- The last two digits indicate publication order within the volume using a Base 36 numbering system employing both numerals and letters. These two-number sets start with 00, 01, 02, 03, 04, 05, 06, 07 , $08,09,0 \mathrm{~A}, \mathrm{OB} \ldots \mathrm{OZ}$, followed by 10-1Z, 20-2Z, etc.

The CID number appears on each page of the manuscript. The complete citation is used on the first page, and an abbreviated version on subsequent pages. Numbers in the index correspond to the last two digits of the six-digit CID number. 


\section{Contents}

vii Conference Committee

SESSION 1 INVITED PAPERS ON INTELLIGENT ROBOTICS

753903 The 17th Annual Intelligent Ground Vehicle Competition: intelligent robots built by intelligent students (Invited Paper) [7539-02]

B. L. Theisen, U.S. Army Tank-Automotive Research, Development and Engineering Ctr. (United States)

753904 Engineering robust intelligent robots (Invited Paper) [7539-03]

E. L. Hall, Univ. of Cincinnati (United States); S. M. A. Ali, The Hashemite Univ. (Jordan);

M. Ghaffari, X. Liao, M. Cao, Univ. of Cincinnati (United States)

753905 Different micromanipulation applications based on common modular control architecture (Invited Paper) [7539-04]

R. Sipola, T. Vallius, M. Pudas, J. Röning, Univ. of Oulu (Finland)

\section{SESSION 2 NOVEL PEOPLE TRACKING APPROACHES}

753906 Object tracking by combining detection, motion estimation, and verification (Invited Paper) [7539-05]

O. Sidla, SLR Engineering (Austria)

753907 Recognizing and tracking humans and vehicles using radar [7539-06]

D. Tahmoush, J. Silvious, Army Research Lab. (United States)

753908 On-line measurement of ski-jumper trajectory: combining stereo vision and shape description [7539-07]

T. Nunner, Joanneum Research (Austria); O. Sidla, SLR Engineering (Austria); G. Paar,

B. Nauschnegg, Joanneum Research (Austria)

753909 Object tracking by co-trained classifiers and particle filters [7539-08]

L. Tang, Hewlett-Packard Labs. China (China); S. Li, Beijing Institute of Technology (China);

K. Liu, L. Wang, Hewlett-Packard Labs. China (China)

\section{SESSION 3 AUTONOMOUS ROBOTIC SYSTEMS AND APPLICATIONS}

$75390 \mathrm{~A}$ Teaching and implementing autonomous robotic lab walkthroughs in a biotech laboratory through model-based visual tracking [7539-9]

M. Wojtczyk, Technische Univ. München (Germany) and Bayer HealthCare (United States);

G. Panin, T. Röder, C. Lenz, S. Nair, Technische Univ. München (Germany); R. Heidemann,

C. Goudar, Bayer HealthCare (United States); A. Knoll, Technische Univ. München

(Germany) 
7539 OB Robust pipeline localization for an autonomous underwater vehicle using stereo vision and echo sounder data [7539-10]

G. M. Breivik, S. A. Fjerdingen, Ø. Skotheim, SINTEF (Norway)

7539 OC Flexible inline low-cost inspection station [7539-11]

C.-K. Sung, Fraunhofer-Institut für Informations- und Datenverarbeitung (Germany)

7539 OD LandingNav: a precision autonomous landing sensor for robotic platforms on planetary bodies [7539-12]

A. Katake, C. Bruccoleri, StarVision Technologies, Inc. (United States); P. Singla, Univ. at Buffalo (United States); J. L. Junkins, Texas A\&M Univ. (United States)

7539 OE Real-time 3D environment model for obstacle detection and collision avoidance with a mobile service robot [7539-13]

J. U. Kuehnle, Fraunhofer-Institut für Produktionstechnik und Automatisierung (Germany); M. Danzer, KUKA Roboter GmbH (Germany); A. Verl, Fraunhofer-Institut für Produktionstechnik und Automatisierung (Germany); R. Bischoff, KUKA Roboter GmbH (Germany)

\section{SESSION 4 AUTONOMOUS ROBOTIC DETECTION, TRACKING, AND VEHICLE ASSISTANCE METHODS}

7539 OF Handling of split-and-merge effects and occlusions using feature-based probabilistic data association [7539-14]

M. Grinberg, F. Ohr, Fraunhofer-Institut für Informations- und Datenverarbeitung (Germany)

7539 OG Real-time object detection and tracking in video sequences [7539-15]

F. Dornaika, IKERBASQUE, Basque Foundation for Science (Spain) and Univ. of the Basque Country (Spain); F. Chakik, Lebanese Univ. (Lebanon)

$7539 \mathrm{OH} \quad$ Robust obstacles detection and tracking using disparity for car driving assistance [7539-16] M. Gouiffès, A. Patri, M. Vasiliu, Univ. Paris-Sud 11 (France)

7539 Ol Assessment of image sensor performance with statistical perception performance analysis [7539-17]

S. Franz, Daimler AG (Germany); D. Willersinn, K. Kroschel, Fraunhofer-Institut für Informationsund Datenverarbeitung (Germany)

$75390 \mathrm{~J} \quad$ Modeling of radial asymmetry in lens distortion facilitated by modern optimization techniques [7539-18]

J. P. de Villiers, Council for Scientific and Industrial Research (South Africa); F. W. Leuschner,

R. Geldenhuys, Univ. of Pretoria (South Africa)

\section{SESSION 5 INTELLIGENT GROUND VEHICLE COMPETITION}

7539 OK The design and results of an algorithm for intelligent ground vehicles [7539-19] M. Duncan, J. Milam, C. Tote, R. N. Riggins, Bluefield State College (United States)

7539 OL Improved single-camera stereo system for mobile robotics [7539-20] W. P. Lovegrove, P. D. McGary, K. Austin, Bob Jones Univ. (United States) 
7539 OM A path planning algorithm for lane-following-based autonomous mobile robot navigation [7539-21]

Y. Aljeroudi, M. Paulik, M. Krishnan, C. Luo, Univ. of Detroit Mercy (United States)

7539 ON Argos: Princeton University's entry in the 2009 Intelligent Ground Vehicle Competition [7539-22]

S. O. Abiola, C. A. Baldassano, G. H. Franken, R. J. Harris, B. A. Hendrick, J. R. Mayer,

B. A. Partridge, E. W. Starr, A. N. Tait, D. D. Yu, T. H. Zhu, Princeton Univ. (United States)

753900 Application of a distributed systems architecture for increased speed in image processing on an autonomous ground vehicle [7539-23]

A. A. Wright, O. Momin, Y. H. Shin, R. Shakya, K. Nepal, D. J. Ahlgren, Trinity College (United States)

7539 OP An enhanced dynamic Delaunay triangulation-based path planning algorithm for autonomous mobile robot navigation [7539-24]

J. Chen, C. Luo, M. Krishnan, M. Paulik, Y. Tang, Univ. of Detroit Mercy (United States)

$7539 \mathrm{OQ}$ Auto-preview camera orientation for environment perception on a mobile robot [7539-25] M. Radovnikovich, P. K. Vempaty, K. C. Cheok, Oakland Univ. (United States)

7539 OR Predictive vision from stereo video: robust object detection for autonomous navigation using the Unscented Kalman Filter on streaming stereo images [7539-26]

D. Rosselot, M. Aull, E. L. Hall, Univ. of Cincinnati (United States)

SESSION 6 AUTONOMOUS ROBOTIC NAVIGATION, SCENE CONTENT, AND CONTROL

7539 OS Synchronizing real and predicted synthetic video imagery for localization of a robot to a 3D environment [7539-27]

D. M. Lyons, S. Chaudhry, Fordham Univ. (United States); D. P. Benjamin, Pace Univ. (United States)

7539 OT Comparison of three control methods for an autonomous vehicle [7539-28]

A. Deshpande, K. Mathur, E. Hall, Univ. of Cincinnati (United States)

\section{SESSION 7 COMPUTER VISION ADVANCES FOR INTELLIGENT ROBOTS}

7539 oU $\quad \mathrm{N}$-dimension closeness measurements used in dynamic pattern recognitions [7539-29]

C. J. Hu, Univ. of Colorado at Boulder (United States)

7539 OV Color image processing for date quality evaluation [7539-30]

D. J. Lee, J. K. Archibald, Brigham Young Univ. (United States)

7539 OW Fast correspondence of unrectified stereo images using genetic algorithm and spline representation [7539-31]

B. Tippetts, D. J. Lee, J. Archibald, Brigham Young Univ. (United States)

7539 OX Color DoG: a three-channel color feature detector for embedded systems [7539-32]

S. Fowers, D. J. Lee, D. K. Wilde, Brigham Young Univ. (United States) 
INTERACTIVE PAPER AND SYMPOSIUM DEMONSTRATION SESSION

$75390 Z$ Active visual tracking method self-adapting to illumination based on particle filter pre-location [7539-34]

J. Su, Harbin Engineering Univ. (China) and Harbin Univ. of Science and Technology (China);

G. Yin, Harbin Engineering Univ. (China); Z. Wei, North China Electronic Power Univ. (China);

Y. Xie, Harbin Univ. of Science and Technology (China)

Author Index 


\title{
Conference Committee
}

\author{
Symposium Chair \\ Jan P. Allebach, Purdue University (United States) \\ Symposium Cochair
}

Sabine Süsstrunk, Ecole Polyłechnique Fédérale de Lausanne

(Switzerland)

Conference Chairs

David P. Casasent, Carnegie Mellon University (United States)

Ernest L. Hall, University of Cincinnati (United States)

Juha Röning, University of Oulu (Finland)

Program Committee

Peter (Ming) Cao, Consultant (United States)

Norbert Lauinger, CORRSYS 3D Sensors AG (Germany)

Dah Jye Lee, Brigham Young University (United States)

Kurt S. Niel, Fachhochschule Wels (Austria)

Yoshihiko Nomura, Mie University (Japan)

Greg Pearly, BAE Systems (United States)

Wolfgang Pölzleitner, Sensotech GmbH (Austria)

Daniel Raviv, Florida Atlantic University (United States)

Neelima Shrikhande, Central Michigan University (United States)

Oliver Sidla, SLR Engineering (Austria)

Bernard L. Theisen, U.S. Army Tank-Automotive Research, Development and Engineering Center (United States)

Dili Zhang, Monotype Imaging (United States)

Session Chairs

1 Invited Papers on Intelligent Robotics

Ernest L. Hall, University of Cincinnati (United States)

2 Novel People Tracking Approaches

Oliver Sidla, SLR Engineering (Austria)

3 Autonomous Robotic Systems and Applications

Juha Röning, University of Oulu (Finland) 
4 Autonomous Robotic Detection, Tracking, and Vehicle Assistance Methods

Ernest L. Hall, University of Cincinnati (United States)

5 Intelligent Ground Vehicle Competition

Bernard L. Theisen, U.S. Army Tank-Automotive Research, Development and Engineering Center (United States)

6 Autonomous Robotic Navigation, Scene Content, and Control

Ernest L. Hall, University of Cincinnati (United States)

Juha Röning, University of Oulu (Finland)

7 Computer Vision Advances for Intelligent Robots

Dah Jye Lee, Brigham Young University (United States) 